\title{
ELASTIC AND PLASTIC ANISOTROPY EFFECTS ON SECOND ORDER INTERNAL STRESSES IN TEXTURED POLYCRYSTALLINE MATERIALS
}

J. KRIER*, H. RUPPERSBERG*, M. BERVEILLER**, P.LIPINSKI**

* Werkstoffwissenschaften, Universität D6600 Saarbrücken, W-Germany

** L.P.M.M.-I.S.G.M.P., F-57045 Metz, France

\section{INTRODUCTION}

The evaluation by X-rays of the residual stress in polycrystalline specimens is based on the determination of the lattice constant a as a function of $\psi[1] . \psi$ is the angle between the normal to the specimen surface and the normal $\mathrm{N}^{\mathrm{h}}$ to the reflecting lattice planes (hkl). For unstrained samples $\mathbf{a}=\mathbf{a}_{\mathbf{o}}$ will be independent of $\psi$.

Stickforth [2] studied the elastic behaviour of statistically, i.e. macroscopically, homogeneous and isotropic media starting from linear relations between all combinations of local and average stress- and strain-tensor components. Neglecting surface anisotropy and $\sigma_{3 \mathrm{i}}$ - terms, he obtained for the normal strain in $\phi-\psi$ direction, i.e. $\varepsilon_{\phi \psi}$, the well known linear $\sin ^{2} \psi$-law :

$$
\varepsilon_{\phi \psi}(h)=\left(a_{\phi \psi}(h) / a_{o}\right)-1=1 / 2 S_{2}(h) \sigma_{\phi} \sin ^{2} \psi+S_{1}(h)\left(\sigma_{11}+\sigma_{22}\right)
$$

(h) stands for (hkl). S1(h) and 1/2 S2(h) are the X-ray elastic constants. $\phi$ is the angle between the 1-direction (which is parallel to the surface) and the projection of $\mathrm{N}^{\mathrm{h}}$ onto the surface. $\sigma_{\phi}=\sigma_{11} \cos ^{2} \phi+\sigma_{22} \sin ^{2} \phi+\sigma_{12} \sin 2 \phi$ corresponds to the normal stress pointing in this direction. Strictly speaking, the $\sigma$-values are volume-averages which are weighted with respect to the absorption of the X-rays of the corresponding local values inside the investigated phase. $\varepsilon_{\phi \psi}(\mathrm{h})$ is the corresponding volume-average of the grains which reflect in the $\phi-\psi$ direction.

Equation (1) fails if the above mentioned conditions are not fulfilled. Much work was done to take into account the influence of crystallographic texture. According to Brak man [3] and to Barral and Sprauel [4] this is possible on the basis of either the Voigt or the Reuss model starting from the Orientation Distribution Function. Depending on the special case, the $\varepsilon\left(\sin ^{2} \psi\right)$ curves may become wavy, just changed in slope or not affected at all. An influence of grain-shape has not yet been studied. Ruppersberg et al. [5] tentatively attributed part of the non-regular behaviour of $\varepsilon\left(\sin ^{2} \psi\right)$-curves obtained for different lattice planes of a cold-rolled nickel plate to the gliding on selected crystal lographic slip systems. Qualitatively, such phenomena were discussed as early as 1923 by Masing and Heyn [6]. However we are not aware of any other theoretical or experimental investigations conducted during the last decades.

In the present paper we study theoretically the elastoplastic behaviour of single-phase polycrystalline specimens by solving the corresponding integral-equations for several 
thousand grains, and by taking the corresponding elastoplastic constitutive-equations into account. We obtain quantitative informations about the average stresses $\sigma$ inside the individual grains which is subdivided into the macroscopic first order stress $\sigma^{\mathrm{I}}$ and a corresponding deviation $\sigma^{I I}$, the second order internal stress. We study the dependence of these quantities on crystallographic and morphologic texture as well as their variation with plastic deformation.

The calculations are performed either for elastically anisotropic cubic crystals (Nickel) or for elastically isotropic but plastically anisotropic FCC crystals.

\section{FORMALISM}

We follow a formalism which was proposed by Berveiller and Zaoui [7] and by $\mathrm{Li}$ pinski and Berveiller [8] for studying the elastoplastic behaviour of micro inhomogeneous media. In this paper, only the static part of the theory, i.e. the relation between total strain and plastic strain is presented. In the dynamic part of the theory, the plastic strain field is calculated as a function of the loading path.

Starting from the equilibrium and compatibility conditions for the local stress $\sigma(r)$ and the total strain $\varepsilon^{t}(r)$, from the linear relation $\sigma(r)=c(r) \varepsilon^{e}(r)$ between the stress and the elastic strain $\varepsilon^{e}(r)$, and by introducing a homogeneous reference medium with elastic constants $\mathrm{C}^{\circ}$, the total strain field $\varepsilon^{\mathrm{t}}(\mathrm{r})$ is given by

$$
\varepsilon_{l m}^{t}(r)=E_{l m}^{t}-\int_{V^{\prime}} \Gamma_{l m i j}\left(r-r^{\prime}\right)\left\{\delta C_{i j k l}\left(r^{\prime}\right) \varepsilon_{k l}^{t}\left(r^{\prime}\right)-C_{i j k l}^{o} \varepsilon_{k l}^{p}\left(r^{\prime}\right)\right\} d V^{\prime}
$$

where

$\mathrm{E}^{\mathrm{t}}$ is the externally applied strain,

$\delta \mathrm{C}(\mathrm{r})=\mathrm{c}(\mathrm{r})-\mathrm{C}^{\circ}$,

$\varepsilon^{\mathrm{P}}(\mathrm{r})=\varepsilon^{\mathrm{t}}(\mathrm{r})-\varepsilon^{\mathrm{e}}(\mathbf{r})$ is the plastic or inelastic strain and

$\Gamma_{\text {lmij }}$ is the modified Green tensor [9] related to the Green tensor $G$ by

$\Gamma_{\text {lmij }}=1 / 2\left(G_{l j, m i}+G_{m j, l i}\right)$.

The specimen consists of $N$ grains $J$ with crystallographic orientations $\Omega^{\mathrm{J}}$ and volume $\mathrm{V}^{\mathrm{J}}$. They are supposed to be homogeneous with respect to the stress $\sigma^{\mathrm{J}}$ and the strains $\varepsilon^{\mathrm{eJ}}, \varepsilon^{\mathrm{pJ}}$. The interaction between two grains $\mathrm{J}$ and $\mathrm{K}$ is described by

$$
T_{l m i j}^{I K}=\frac{1}{V_{J}} \int_{V^{J}} \int_{V^{K}} \Gamma_{l m i j}\left(r^{J}-r^{K}\right) d V^{J} d V^{K}
$$

For $\mathrm{J} \neq \mathrm{K}$, the self consistent approximation of $\mathrm{J}$ imbedded in the effective homogeneous matrix with $\mathrm{C}^{\circ}=\mathrm{C}^{\text {eff }}$ is used and one obtains

$$
\varepsilon_{l m}^{e J}=E_{l m}^{t}-\varepsilon_{l m}^{p J}-T_{l m i j}^{I J}\left(C_{i j k l}^{\text {eff }} \varepsilon_{k l}^{p J}-\delta C_{i j k l}^{J} \varepsilon_{k l}^{t J}\right)
$$


which allows to calculate $\varepsilon^{\mathrm{eJ}}$ and $\sigma^{\mathrm{J}}$ in every grain $\mathrm{J}$ for a given external load and plastic strain field $\varepsilon^{\mathrm{pJ}} . \varepsilon^{\mathrm{eJ}}$ depends of the shape and the orientation of the grain $\mathrm{J}$ and of the microstructure (crystallographic and morphological texture) of the medium. These parameters have some influence on the $C^{\text {eff }}$ which is also calculated according to the self consistent approximation.

We call $\mathrm{N}_{\mathrm{i}}^{\mathrm{h}}(\cos \varphi \sin \psi, \sin \varphi \sin \psi, \cos \psi)$ the components of $\mathrm{N}^{\mathrm{h}}$ defined in the in troduction for the grain J. The normal elastic strain $\varepsilon^{\beta}{ }_{\varphi \psi}$ pointing in the direction $N^{\text {h }}$ is given by $\varepsilon^{\mathrm{eJ}}{ }_{\mathrm{ij}} \mathrm{N}_{\mathrm{i}}^{\mathrm{h}} \mathrm{N}_{\mathrm{j}}^{\mathrm{h}}$. $\beta$ is explained below.

Neglecting absorption effects, $\varepsilon_{\varphi \psi}(\mathrm{h})$ is obtained from

$$
\varepsilon_{\varphi \psi}(h)=\sum_{N} v^{J} \varepsilon_{i j}^{e J} N_{i}^{h} N_{j}^{h} / \sum_{N} v^{J}
$$

where the sums have to be taken over all grains which satisfy Bragg's law. One degree of freedom is left for the orientation of these grains, namely the angle $\beta$ by which they are rotated about $N^{h}$. In general, $\sigma^{J}$ varies considerably with $\beta$ giving rise to line broa dening and to effects of texture on the $\varepsilon\left(\sin ^{2} \psi\right)$ law (Eq. 1).

\section{APPLICATIONS}

We first studied the purely elastic behaviour (i.e. the variation of $\varepsilon^{\mathrm{J}}$ due to an applied external stress $\sigma^{I}$ which does not yield any additional plastic deformation) of a specimen consisting of 1000 equal-size grains. Each grain behaves in an elastically anisotropic manner corresponding to single crystals of nickel. In all figures, the $\varepsilon^{\beta}{ }_{\varphi \psi}$ values of all reflecting grains are given as a function of $\sin ^{2} \psi$. A spread of the points for a given $\sin ^{2} \psi$ value is due to the different $\beta$ angles of the corresponding grains.

For an external load $\sigma_{11}^{I}=100 \mathrm{MPa}$, Fig. 1 shows the results obtained for spherical grains with isotropic and anisotropic orientation-distributions. The latter corresponds to a texture resulting from $100 \%$ plastic deformation by rolling. For the (422) plane, the external load leads to a cloud of $\varepsilon^{\beta} 0 \psi$-points which scatter about the solid line obtained by integrating over $\beta$ and which therefore corresponds to the experimentally obtained $\varepsilon_{0} \psi\left(\sin ^{2} \psi\right)$ curve. The mean squared deviation of the points from the line increases with $\sigma^{I}$ and the same will happen with the width of the diffraction peaks. In the textured material, the $\varepsilon_{0 \psi \text {-points are seen to be no longer uniformly distributed over }}$ $\beta$. The solid line becomes wavy and the $\sigma^{I}$-induced linebroadening decreases. The results shown for the (200) and the (111) planes confirm the observation by Dölle [10] that the normal $\varepsilon_{\varphi \psi}$ strains which are perpendicular to the (h00) and (hhh) planes do not depend on $\beta$. Therefore, the individual points lie on straight lines. There is no $\sigma^{I}$ induced line broadening and texture affects only the slope of the linear $\varepsilon\left(\sin ^{2} \psi\right)$ curves. Fig. 2 demonstrates the influence of grain shape anisotropy. The specimen has no crystallographic texture, but all grains have the same shape of prolate spheroïds with $a_{1}$ in the 1-direction being ten times larger than $a_{2}$ and $a_{3}$. The resulting $\varepsilon^{\beta_{0 \psi}}$ points clearly deviate from the positions in Fig. 1 obtained with spherical grains. The deviation from the mean $\varepsilon_{\varphi \psi}\left(\sin ^{2} \psi\right)$ curves of spherical grains, which are plotted as solid lines in Fig. 2, is largest for the (200) reflection. This is related to the fact that the difference between the limiting Reuss and Voigt cases is strongest for this orientation [1]. The ef- 
between the limiting Reuss and Voigt cases is strongest for this orientation [1]. The effect of grain shape and crystallographic anisotropy may therefore clearly be distinguished.

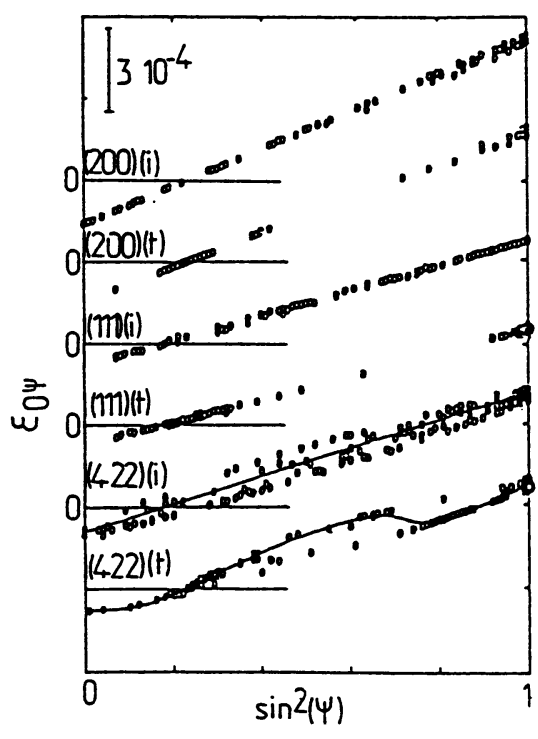

Fig. $1 \varepsilon^{\beta} 0 \psi\left(\sin ^{2} \psi\right)$ values of the reflecting spherical grains (points) and mean $\varepsilon_{0 \psi}\left(\sin ^{2} \psi\right)$ curves for isotropic (i) and textured $(t)$ specimens. The single crystal properties correspond to $\mathrm{Ni}$, $\sigma_{11}^{I}=100 \mathrm{MPa}$.

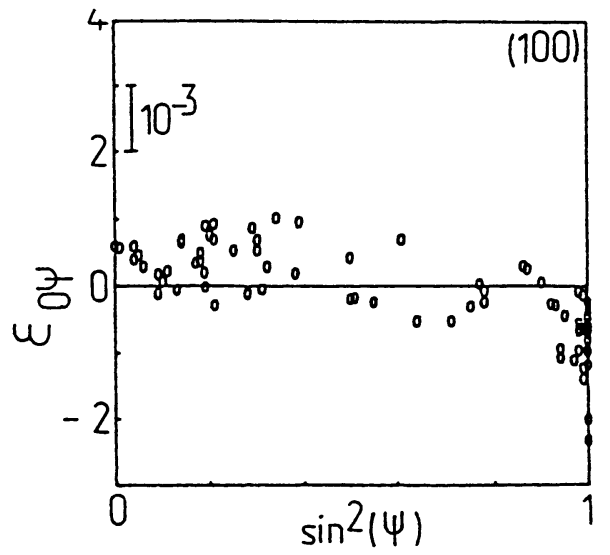

$$
\sigma_{11}=100 \mathrm{MPa} \text {. }
$$

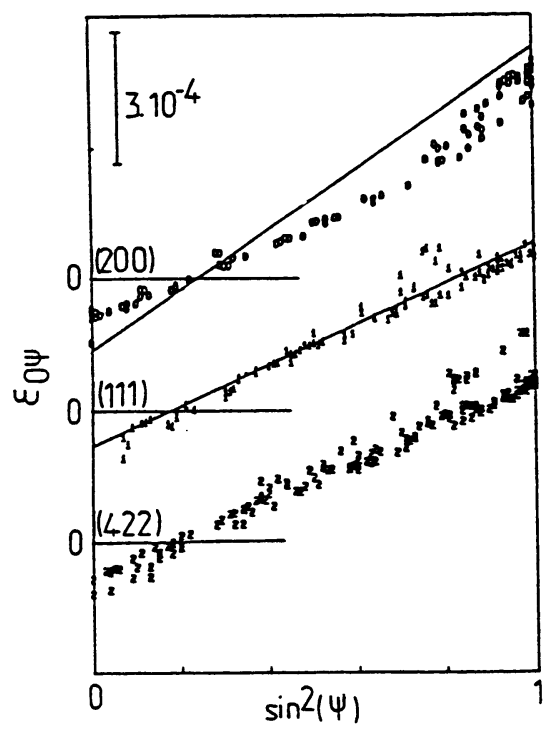

Fig. $2 \varepsilon^{\beta} 0 \psi\left(\sin ^{2} \psi\right)$ values of the reflecting elongated grains (points) and for spherical grains (solid lines). (Ni single crystals and $\sigma_{11=100 \mathrm{MPa}) \text {. }}$

Fig. $3 \varepsilon^{\beta} 0 \psi\left(\sin ^{2} \psi\right)$ values of the reflecting elastically isotropic grains after $20 \%$ plastic deformation of the specimen. 
For studying "Heyn stresses", i.e. $\sigma^{\mathrm{II}}$ related to the incompatibility of plastic deformation, we investigated 5000 elastically isotropic $\left(v=0.3, \mu=3.10^{4} \mathrm{MPa}\right)$ grains which initially had spherical shape and random orientations. Gliding was allowed to take place on the usual $24<111>(100)$ slip systems according to Schmid's law with an initial critical yield strength of $\tau^{\circ}=140 \mathrm{MPa}$. The strain hardening was taken into account by a $24 \times 24$ hardening matrix [11] consisting of a first term equal to $\mu / 250$ which caracterizes the usual interactions and a second one which is $50 \%$ larger and corresponds to the strong interactions between dislocations.

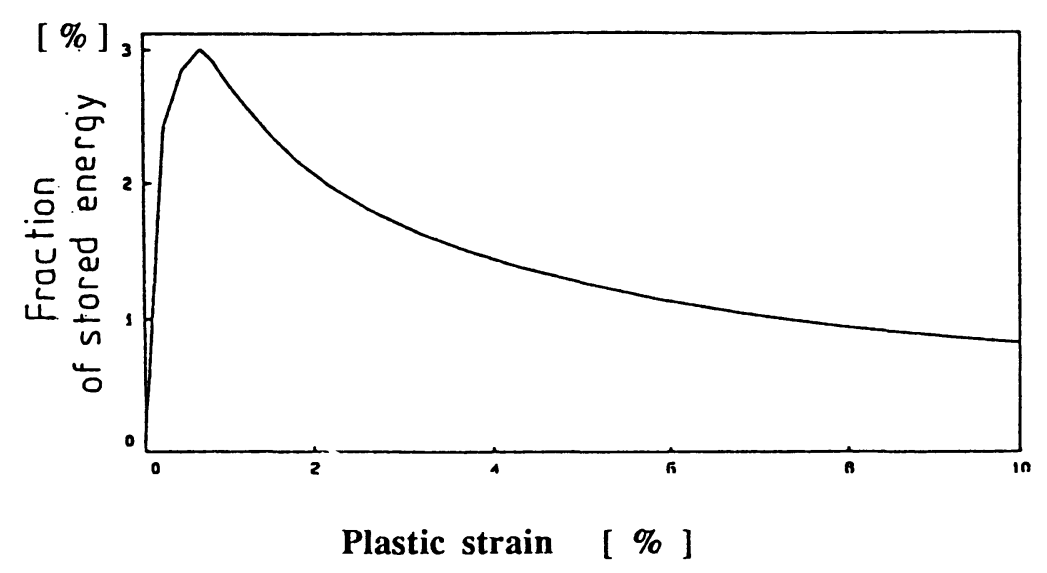

Fig. 4 Fraction of the stored energy as a function of uniaxial plastic deformation.

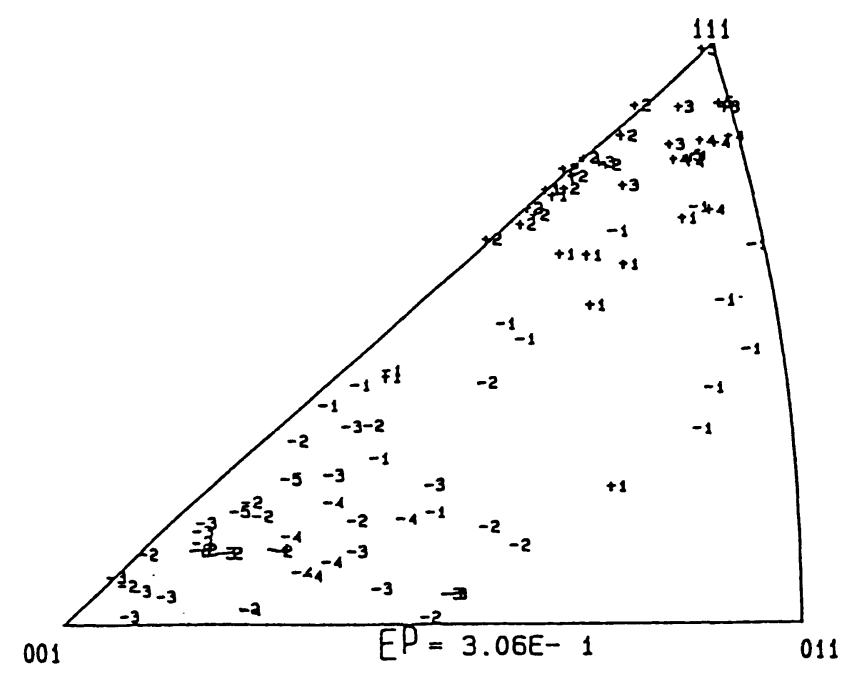

Fig. 5 Inverse pole figure in 1-direction of the amplitude a of $\sigma^{\mathrm{II}} 11$ after $30 \%$ uniaxial deformation in 1-direction. The stress component is given by: $5(\mathrm{a}-1)<\sigma<5 \mathrm{a}$ for $\mathrm{a}>0$ and $5(\mathrm{a}+1)>\sigma>5 \mathrm{a}$ for $\mathrm{a}<0$. [ MPa ] 
Rolling was simulated by applying a load $\sigma_{11}=-\sigma_{33}$, whereas the other components $\sigma_{i j}$ remain zero, until a plastic deformation of $20 \%$ was reached. After unloading $\left(\sigma^{\mathrm{I}}=0\right), \sigma^{\mathrm{II}}$ stresses remained in the individual grains which are due to the incompatibility of the $\varepsilon^{p_{i j}}(r)$ field. The plots of $\varepsilon^{\beta} 0 \psi$ vs. $\sin ^{2} \psi$ for the (111) and (100) planes are shown in Fig. 3.

The widths of the diffraction peaks will be increased and strong modulations of the average $\varepsilon_{\varphi \psi}$ vs. $\sin ^{2} \Psi$ curves occur. As seen in Fig. 3, these effects strongly depend on the reflecting planes as postulated by Greenough [12]. Additionally these figures indicated the formation of crystallographic texture. To check whether these high $\sigma^{\text {II }}$ values make sense, we calculated the corresponding fraction of the stored energy [11] which is given in Fig. 4 and which compares well with experimental observations [13] [14].

Finally we give in Fig. 5, the inverse pole figure of the tensile direction 1 of a specimen after a $30 \%$ plastic deformation. In this figure the amplitude of the $\sigma^{\text {II }}{ }_{11}$ residual stresses are indicated which again aggree well with experimental observations on single crystal behaviour : The crystals with $<111>$ orientation harden more strongly than those with $<100>$ parallel to tensile axis. As a consequence the residual stresses in $<111>$ crystals are positive.

\section{ACKNOWLEDGEMENTS}

Financial supports of E.C. and PROCOPE are gratefully acknowledged.

\section{REFERENCES}

1. I.C. Noyan and J.B. Cohen, Residual stress Measurement by Difraction and Interpretation, Springer Verlag (1987)

2. J. Stickforth, Techn. Mitt. Krupp, 69, 24 (1966)

3. C.M. Brakman, J. Appl. Cryst., 16, 24 (1983)

4. M. Barral and J.M. Sprauel, Eigenspannungen Eds Macherauch, Hauk DGM Ober wisel, 2, 31 (1983)

5. H. Ruppersberg, I. Detemple and J. Krier, Phys. Stat. Sol (a), 116, 681 (1989)

6. G. Masing and E. Heyn, Wiss. Veröff. Siemens-Konz 3, 231 (1923) and 5, 135 (1926)

7. M. Berveiller and A. Zaoui, J. of Engng Mat. and Technology, 106, 295 (1984)

8. P. Lipinski and M. Berveiller, Int. J. of Plasticity, 5, 149 (1989)

9. E. Kröner, J. Engng. Mech. Div. Proc. ASCF, 106, 889(1980)

10. H. Dölle, J. Applied Cryst., 12 , 489 (1979)

11. P. Lipinski, J. Krier and M. Berveiller, Revue Phys. Appl., 25, 361 (1990)

12. G.B. Greenough, Progr. in Met. Phys., 3 (1952)

13. M.B. Bever, D.L. Holt, A.L. Titchener, Prog. Mat. Sciences, 17, 1 (1973)

14. A. Chrysochoos, Thèse d'Etat, université de Montpellier (1987) 